\title{
Beamstrahlung for a longitudinally non-uniform bunch
}

\section{Citation}

Jacob, M., and Tai Tsun Wu. 1989. "Beamstrahlung for a Longitudinally Non-Uniform Bunch." Nuclear Physics B 318 (1): 53-74. https://doi.org/10.1016/0550-3213(89)90047-3.

\section{Permanent link}

http://nrs.harvard.edu/urn-3:HUL.InstRepos:41555800

\section{Terms of Use}

This article was downloaded from Harvard University's DASH repository, and is made available under the terms and conditions applicable to Other Posted Material, as set forth at http:// nrs.harvard.edu/urn-3:HUL.InstRepos:dash.current.terms-of-use\#LAA

\section{Share Your Story}

The Harvard community has made this article openly available.

Please share how this access benefits you. Submit a story.

\section{Accessibility}




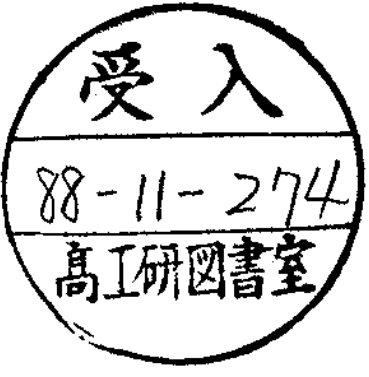

CERN-TH. 5192/88

BEAMSTRAHLUNG FOR LONGITUDINALLY NON-UNIFORM BUNCH

M. Jacob

CERN - Geneva

and

$\operatorname{Tai}$ Tsun $\mathrm{Wu}^{*}$ )

CERN - Geneva

and

Gordon McKay Laboratory, Harvard University

\section{A B S T R A C T}

Bremsstrahlung energy loss by electrons and positrons in very high energy $\mathrm{e}^{+} \mathrm{e}^{-}$linear colliders are calculated systematically in terms of Feynman diagrams for the case of longitudinally non-uniform bunches. The radiation rate is given as a sum of three texms, which include not only the known leading contributions but also the end and non-uniformity effects.

*) Work supported in part by the United States Department of Energy under grant DE-FG02-84ER40158. 


\section{1. - INTRODUCTION}

With circular electron-positron colliders, it is virtually impossible to get beyond centre-of-mass energies of about $300 \mathrm{GeV}$, the limiting factor being synchrotron radiation. This problem can be avoided by constructing linear electron-positron colliders. However, because of the necessary very high charge densities in the bunch in order to achieve useful luminosity, there is intense bremstrahlung during bunch crossing. In a recent series of papers [1-3], we have studied this bremsstrahlung process, referred to as beamstrahlung [4], using the natural approach of Feynman diagrams. The conditions considered are typical of those relevant for $\mathrm{e}^{+} \mathrm{e}^{-}$linear colliders in the multi-TeV régime [5]. Alternative approaches not using Feynman diagrams have also been carried out, yielding simflar results $[6,7]$. All these analytic approaches so fax use the approximation low-disruption factor.

In all these considerations $[1-3,6,7]$, the charge density inside the bunch is assumed to be unfform. While this assumption simplifies greatly the calculation, it is far fxom being realistic. Recently, attempts [8-11] have been made to study longitudinally non-unfform bunches. In [10] and [11], only "almost uniform" bunches were considered. In [8] and [9], the authors extrapolated their results to non-uniform bunches, reaching the conclusion that the effect of non-uniformity on beamstrahlung is large. However, this conclusion $[8,9]$ appears to be of dubious validity.

It is the purpose of the present paper to give a systematic approach to the case of longitudinally non-uniform bunch, using again the method of Feynman diagrams [1-3]. A typical realistic longitudinal charge distribution is the hyperbolic secant distribution [12]

$$
\operatorname{sech}^{2}\left(\frac{1}{2} z / L_{b}\right)
$$

where $\mathrm{L}_{b}$ is the nominal bunch length. Such a distribution is not "almost uniform"; Indeed it describes how the density goes to zero away from the centre of the bunch. The considerations presented here are of course not limited to this special form (1.1).

Our results are to be found in Section 11 . 


\section{2. - METHOD OF APPROACH}

The present study is technically difficult, much more so than any of the previous papers on beamstrahlung [1-4,6-11]. In this section, we give a brief description of the method to be followed.

As discussed in [1], there are three important length scales. They are respectively $\mathrm{L}_{c}$, the correlation length or the distance travelled by the electron or positron near the longitudinal density maximum for a deflection angle of $\mathrm{m} / \mathrm{E}$, $L_{b}$, the bunch length, and $L_{e}$, the virtual electron length. Actually, instead of $L_{c}$, the more relevant length is [1]

$$
l_{c}=\left(L_{c}^{2} L_{e}\right)^{1 / 3},
$$

which is independent of the electron mass. This new correlation length $\ell_{c}$ applies to realistic machines in the $\mathrm{TeV}$ range with beamstrahlung radiation in the deep quantum regime. In practice, the regime of interest is

$$
\ell_{c} \ll L_{b} \ll L_{e} .
$$

Assuming the disruption factor to be small, we can approximate the bunch-bunch scattering by that of an electron (positron) off the positron (electron) bunch. Thus the process to be studied is, for example,

$$
e^{-}+\text {bunch } \longrightarrow e^{-}+\gamma+\text { bunch, }
$$

described by the Feynman diagram shown in Fig. 1. Using the high-energy approximation developed thirty years ago [13,14], the matrix element for this Feynman diagram can be written down explicitly.

In Section 3, we take this matrix element, calculate its modulus square and then integrate over the final txansverse momenta to obtain the photon energy distribution. This development is similar to that of [11].

In ordex to study systematically the photon energy distribution without a priori knowledge of the result, we find it difficult to avoid using the method of Mellin transform [15]. This approach is carried out in Section 4. For the present problem, the more recently developed version of Mellin transform [16] is fortunately not needed.

In this approach, using Mellin transform, the desired terms that describe beamstrahlung are obtained successively from the residues at $\zeta=1, \zeta=0$ and 
$\zeta=-1$, where $\zeta$ is the Mellin-transform variable. For this purpose, explicit analytic continuation is of central importance. The steps are:

i) extraction of the residue at $\zeta=1$;

ii) analytic continuation from $\operatorname{Re} \zeta>1$ to $\operatorname{Re} \zeta>0$ by deforming a contour of integration:

iii) extraction of the residue at $\zeta=0$;

iv) analytic continuation from $\operatorname{Re} \zeta>0$ to $\operatorname{Re} \zeta>-1$ by deforming another contoux of integration in a different variable; and

v) extraction of the residue at $\zeta=-1$.

These five steps are carried out respectively in Sections 5-9.

The result consists of three terms. Roughly speaking, two of the terms, the one from the residue at $\zeta=1$ and a part of the one from that at $\zeta=0$, are generalizations of the previously known texms from the longitudinally uniform bunch. The rest of the term from the residue at $\zeta=0$ describes the end effect of the bunch. Finally, the third term, from the residue at $\zeta=-1$, describes the effect of charge density gradiant. This last term bears similarity to those obtained in [8-11]; however, it is now in the form of a convergent integral and it is not especially large numerically.

In Section 10, as an illustration, the result is applied to the hyperbolic secant distribution $(1.1)$.

\section{3. - ENERGY DISTRIBUTION OF BEAMSTRAHLUNG PHOTON}

The charge distribution in the bunch is assumed to be longitudinally non-uniform but transversely uniform within a cixcle of radius $R$. Let $N$ denote the total number of positrons (or electrons) in a bunch, and $L_{b}$ the nominal bunch length, then the average charge density is

$$
\frac{N e}{\pi L_{b} R^{2}} \text {. }
$$

Let this bunch length $\mathrm{L}_{b}$ be used as the scale along the bunch, taken to be in the $z$ direction, then we define a normalized charge density $\rho$ by expressing the actual charge density $\rho_{0}$ as

$$
P_{0}(z)=\frac{N e}{\pi L_{b} R^{2}} P\left(\frac{z}{L_{b}}\right)
$$

With (3.1), the potential term eV in the Klein-Gordon and the Dirac equations is given by

$$
\text { e } V(r, z)=\frac{N \alpha}{L_{b}} \frac{r^{2}}{R^{2}} \rho\left(\frac{z}{L_{b}}\right) \text {, }
$$


where the additional logarithmic term is neglected [2].

In view of the form of the phase for the initial and final state wave functions [14], we need to define

$$
\begin{aligned}
& \tau(\xi)=\int_{-\infty}^{\xi} d \xi^{\prime} p\left(\xi^{\prime}\right), \\
& T(\xi)=\int_{-\infty}^{\xi} d \xi^{\prime}\left[\tau\left(\xi^{\prime}\right)\right]^{2}, \\
& U(\xi)=\int_{-\infty}^{\xi} d \xi^{\prime} \tau\left(\xi^{\prime}\right), \\
& \tau_{f}(\xi)=\int_{\xi}^{\infty} d \xi^{\prime} p\left(\xi^{\prime}\right), \\
& T_{f}(\xi)=\int_{\xi}^{\infty} d \xi^{\prime}\left[\tau_{f}(\xi)\right]^{2}, \\
& U_{f}(\xi)=\int_{\xi}^{\infty} d \xi^{\prime} \tau_{f}(\xi),
\end{aligned}
$$

and

$$
W\left(\xi, \xi^{\prime}\right)=T(\xi)-T\left(\xi^{\prime}\right)-\left(\xi-\xi^{\prime}\right)^{-1}\left[U(\xi)-U\left(\xi^{\prime}\right)\right]^{2}
$$

Because of the way $\rho(\xi)$ is normalized, $\tau(\xi)$ has the propexty that $\tau(\xi) \rightarrow 1$ as $\xi \rightarrow \infty$. This implies a simple relation between $\tau(\xi)$ and $\tau_{f}(\xi)$, namely

$$
\tau(\xi)+\tau_{f}(\xi)=1
$$

The corresponding relations between $T(\xi)$ and $T_{f}(\xi)$, and between $U(\xi)$ and $U_{f}(\xi)$, are more complicated. However, the $W$ of (3.9) can be expressed equally well in terms of $\mathrm{T}_{\mathrm{f}}$ and $\mathrm{U}_{\mathrm{f}}$ :

$$
W\left(\xi, \xi^{\prime}\right)=-T_{f}(\xi)+T_{f}\left(\xi^{\prime}\right)-\left(\xi-\xi^{\prime}\right)^{-1}\left[U_{f}(\xi)-U_{f}\left(\xi^{\prime}\right)\right]^{2} \text {. }
$$

Besides the obvious symmetxy

$$
W\left(\xi^{\prime}, \xi\right)=-W\left(\xi, \xi^{\prime}\right),
$$

W has one more interesting property. It follows from the Schwarz inequality 


$$
\left[\int_{\xi^{\prime}}^{\xi} d \xi^{\prime \prime} \tau\left(\xi^{\prime \prime}\right)\right]^{2} \leqslant\left\{\int_{\xi^{\prime}}^{\xi} d \xi^{\prime \prime}\left[\tau\left(\xi^{\prime \prime}\right)\right]^{2}\right\}\left[\int_{\xi^{\prime}}^{\xi} d \xi^{\prime \prime}\right]
$$

that

$$
\left[U(\xi)-U\left(\xi^{\prime}\right)\right]^{2} \leqslant\left(\xi-\xi^{\prime}\right)\left[T(\xi)-T\left(\xi^{\prime}\right)\right]
$$

If the observation is made that the longitudinal charge distribution is nowhere zero inside any realistic bunch, than $\tau(\xi)$ is a strictly increasing function of $\xi$ inside the bunch, and for Eq. (3.14) the equality sign holds only for $\xi=\xi^{\prime}$. Therefore

$$
\operatorname{sign} \text { of } W\left(\xi, \xi^{\prime}\right)=\operatorname{sign} \text { of } \xi-\xi^{\prime} \text {. }
$$

This property (3.15) is of central importance in the development of this paper.

It is now straightforward to write down the matrix element of the Feynman diagram of Fig. 1 for both the Klein-Gordon case of spin 0 [2] and the Dirac case of $\operatorname{spin} \frac{1}{2}[3]$, to take 1 ts modulus square, to sum over the spin in the latter case, neglecting the helicity-flip contribution which is small, and to integrate over the txansverse momenta of the outgoing particles [11] to obtain the energy distribution of the beamstrahlung photon. The result is

$$
\begin{aligned}
& I(X)=\frac{-i \alpha}{2(2 \pi) k_{i}}\left(\frac{R}{2 N \alpha}\right)^{2} s(X) \int_{-\infty}^{\infty} d z \int_{-\infty}^{\infty} d z^{\prime}\left(z-z^{\prime}\right)^{-1}\left[\tau\left(\frac{z}{L_{b}}\right)-\tau\left(\frac{z^{\prime}}{L_{b}}\right)\right]^{2} \\
& \int_{0}^{\left(\frac{2 N \alpha}{R}\right)^{2}} \eta d \eta \exp \left\{i \frac{X}{2(1-X) k_{i}}\left[m^{2}\left(z-z^{\prime}\right)+\eta L_{b} W\left(\frac{z}{L_{b}}, \frac{z^{\prime}}{L_{b}}\right)\right]\right\}_{,}(3.16)
\end{aligned}
$$

where $k_{1}$ is the energy of the electron or positron under consideration, $x$ is the fractional energy of the radiated photon (i.e., the photon energy divided by $k_{1}$ ), $s(X)$ is a spin factor

$$
s(X)=\frac{1}{2} \frac{2-2 X+X^{2}}{1-X}
$$

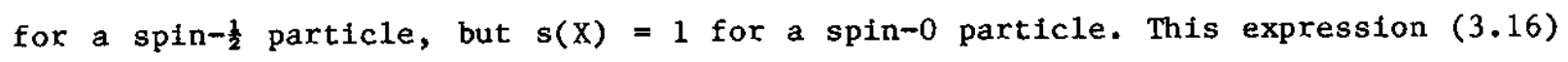
is essentially Eq. (35) with Eq. (36) of [11], except for the additional term $\mathrm{m}^{2}\left(z-z^{\prime}\right)$ here. Remember that we must divide the radiation rate by the beam cross-section $\pi R^{2}$ in order to get $I(X)$. This term $m^{2}\left(z-z^{\prime}\right)$, proportional to the small quantity $\mathrm{m}^{2}$, was neglected in [11] because, in the special case considered thexe, the length $\mathrm{L}_{e}$ did not play a role. In contrast, here $\mathrm{L}_{e}$ is a very important length scale in addition to $L_{b}$ and $l_{c}$. In spite of the appearance of the overall $i$ factor, the right-hand side of (3.16) is of course real. 
The expression ( 3.16 ) actually depends on the quantities $k_{i}$ and $L_{b}$ only through the ratio $\mathrm{L}_{b} / \mathrm{k}_{i}$. It is therefore the same in the laboratory frame as in the frame where the bunch is at rest. To make this explicit, it is only necessary to scale $z$ by $\mathrm{L}_{b}$. If $\eta$ is scaled by $(2 \mathrm{~N} \alpha / R)^{2}$, then

$$
\begin{gathered}
I(X)=\frac{-i \alpha}{\pi} \Lambda^{3} s(X) \int_{-\infty}^{\infty} d \xi \int_{-\infty}^{\infty} d \xi^{\prime}\left(\xi-\xi^{\prime}\right)^{-1}\left[\tau(\xi)-\tau\left(\xi^{\prime}\right)\right]^{2} e^{i \beta\left(\xi-\xi^{\prime}\right)} \\
\qquad \int_{0}^{1} d \eta \eta \exp \left[i \eta \frac{2 X}{1-X} \wedge^{3} W\left(\xi, \xi^{\prime}\right)\right]
\end{gathered}
$$

where

$$
\beta=\frac{X m^{2} L_{b}}{2(1-X) k_{i}}=\frac{X}{1-X} \frac{L_{b}}{L_{e}}
$$

and [1-3]

$$
\Lambda=\left(\frac{N^{2} \alpha^{2} L_{b}}{k_{i} R^{2}}\right)^{1 / 3}=\frac{L_{b}}{l_{c}}
$$

Note that the average fractional beamstrahlung energy loss is

$$
\delta=\int_{0}^{1} d X X I(X) .
$$

With this starting point, we investigate in detail the behaviour of the right-hand side of (3.18) for laxge $\Lambda$.

\section{4. - MELLIN TRANSFORM}

For. the uniform bunch, it has been found that [3]

$$
\delta=\frac{\alpha}{\pi}\left[1.38 \frac{L_{b}}{l_{c}}+\frac{4}{3} \ln \frac{4 L_{e}}{l_{c}}+0(1)\right] .
$$

The two explicit terms on the right-hand side of (4.1) can be assigned to different oxigins: the first term to radiation during bunch crossing, while the second one to radiation before and aftex bunch crossing. In the absence of a sharp edge, such a distinction is not possible in the present case of a non-uniform bunch, such as the hyperbolic secant one $(1,1)$. Nevertheless, it remains that the two conditions $\ell_{c} \ll \mathrm{L}_{b}$ and $\mathrm{L}_{\mathrm{b}} \ll \mathrm{L}_{\mathrm{e}}$ of $(2.2)$ are essentially independent.

Since the asymptotic behavioux of a multiple integral with two large parameters is in general quite difficult to determine [16], we make use of this independence and consider the case 


$$
\ell_{c}<L_{b}
$$

with $\mathrm{L}_{b}$ and $\mathrm{L}_{e}$ comparable in magnitude. For the "super" machine parameters $[1,5,6]$, these lengths are roughly

$$
\ell_{c} \sim 0.05 \mathrm{~m} \quad L_{b} \sim 3 \mathrm{~m} \quad L_{e} \sim 40 \mathrm{~m}
$$

in the bunch frame. Thus $\mathrm{L}_{\mathrm{b}} / \ell_{\mathrm{c}} \sim 60$ while $\mathrm{L}_{\mathrm{e}} / \mathrm{L}_{\mathrm{b}} \sim 13$.

In oxder to determine the asymptotic behaviour of $\mathrm{I}(\mathrm{X})$ with the conditions (4.2), we employ the method of Mellin transform, which has been used to analyze the high-energy behaviour of Feynman diagrams $[15,17]$. The Mellin txansform of (3.18) is to be carried out with respect to the variable $\Lambda$, defined by (3.20). Thus we consider the photon spectrum as a function of $\Lambda$ with the parameter $\mathrm{X}$, rather than a function of $X$ with the parameter $\Lambda$. With this in mind, we write

$$
I(X)=\frac{\alpha}{\pi} s(X) K(\Lambda),
$$

which defines $K(\Lambda)$. Of course, both $I(X)$ and $K(\Lambda)$ are actually functions of both $X$ and $\Lambda$.

With this notation, define the Mellin transform

$$
\bar{K}(\zeta)=\int_{0}^{\infty} d \wedge \Lambda^{-1-\zeta} K(\Lambda)
$$

Since, as seen from (3.18) and (4.1),

$$
K(\Lambda)=O(\Lambda)
$$

as $\Lambda \rightarrow \infty$, and

$$
K(\Lambda)=O\left(\Lambda^{3}\right)
$$

as $\Lambda \rightarrow 0$, the $\bar{K}(\zeta)$ as defined by (4.5) is analytic for

$$
3>\operatorname{Re} 5>1 \text {. }
$$

The residue of $\overline{\mathrm{K}}(\zeta)$ at $\zeta=1$ glves the leading behaviour of $\mathrm{K}(\Lambda)$, and hence of $I(X)$, in the limit $\ell_{c} \ll L_{b}[18]$. This residue is computed in the next section. Highex-oxder terms, including the end and non-uniformity effects, are to be extracted from the behaviours of the analytic continuation of $\overline{\mathrm{K}}(\zeta)$ at $\zeta=0$ and $\zeta=-1-$ see Section 7 and Section 9 , respectively. [The behaviour of $\bar{k}(\zeta)$ near 
$\zeta=3$, on the contraxy, is of no interest. It gives merely the behaviour of $I(X)$ for the opposite 1imit $\left.\ell_{c} \gg L_{b} \cdot\right]$

Remaining in the region (4.8), we can substitute (3.18) into (4.5) to get an explicit expression for $\overline{\mathrm{K}}(\zeta)$. The $\Lambda$ integxal is

$$
\begin{aligned}
\int_{0}^{\infty} d \wedge \Lambda^{-1-\xi} \Lambda^{3} \exp \left[i \eta \frac{2 X}{1-X} \Lambda^{3} W\left(\xi, \xi^{\prime}\right)\right] \\
=\left\{\begin{array}{l}
\frac{1}{3} i e^{-i \pi \xi / 6} \Gamma(1-\xi / 3)\left[\eta \frac{2 X}{1-X} W\left(\xi, \xi^{\prime}\right)\right]^{-1+5 / 3} \text { for } W\left(\xi, \xi^{\prime}\right)>0, \\
-\frac{1}{3} i e^{i \pi \xi / 6} \Gamma(1-\xi / 3)\left[-\eta \frac{2 X}{1-X} W\left(\xi, \xi^{\prime}\right)\right]^{-1+5 / 3} \text { for } W\left(\xi, \xi^{\prime}\right)<0 .
\end{array}\right.
\end{aligned}
$$

Because of (3.15), this can be rewritten as

$$
\begin{aligned}
& \int_{0}^{\infty} d \Lambda \Lambda^{-1-\xi} \Lambda^{3} \exp \left[i \eta \frac{2 X}{1-\bar{X}} W\left(\xi, \xi^{\prime}\right)\right] \\
= & \frac{1}{3} i \operatorname{sg}\left(\xi-\xi^{\prime}\right) e^{-i \pi(5 / 6) \operatorname{sg}\left(\xi-\xi^{\prime}\right)} \Gamma(1-5 / 3)\left[\eta \frac{2 X}{1-X}\left|W\left(\xi, \xi^{\prime}\right)\right|\right]^{-1+5 / 3}
\end{aligned}
$$

The substitution into (4.5) then gives, using (4.4),

$$
\begin{aligned}
\bar{K}(\xi)= & \frac{1}{3} \Gamma(1-\xi / 3)\left(\frac{2 X}{1-X}\right)^{-1+5 / 3} \int_{-\infty}^{\infty} d \xi \int_{-\infty}^{\infty} d \xi^{\prime}\left|\xi-\xi^{\prime}\right|^{-1}\left[\tau(\xi)-\tau\left(\xi^{\prime}\right)\right]^{2} \\
& e^{i \beta\left(\xi-\xi^{\prime}\right)} e^{-i \pi(5 / 6) s g\left(\xi-\xi^{\prime}\right)}\left|W\left(\xi, \xi^{\prime}\right)\right|^{-1+5 / 3} \int_{0}^{1} d \eta \eta^{5 / 3} \\
= & (3+5)^{-1} \Gamma(1-5 / 3)\left(\frac{2 X}{1-X}\right)^{-1+5 / 3} \int_{-\infty}^{\infty} d \xi \int_{-\infty}^{\infty} d \xi^{\prime}\left|\xi-\xi^{\prime}\right|^{-1}\left[\tau(\xi)-\tau\left(\xi^{\prime}\right)\right]^{2} \\
& e^{i \beta\left(\xi-\xi^{\prime}\right)} e^{-i \pi(5 / 6) s g\left(\xi-\xi^{\prime}\right)}\left|W\left(\xi, \xi^{\prime}\right)\right|^{-1+5 / 3}
\end{aligned}
$$

Its behaviours near $\zeta=1,0,-1$ are to be determined in succession.

\section{5. - RESIDUE OF $\overline{\mathrm{K}}(\zeta)$ AT $\zeta=1$}

At $\zeta=1, \bar{K}(\zeta)$ has a simple pole. Let $R_{l}$ be its residue, i.e.,

$$
R_{1}=\lim _{\zeta \rightarrow 1}(5-1) \bar{K}(\zeta) .
$$


The contribution to this residue comes from the vicinity of $\xi=\xi$ '. Let

$$
\vec{\xi}=\frac{1}{2}\left(\xi+\xi^{\prime}\right)
$$

and

$$
\mu=\xi-\xi^{\prime}
$$

For $\mu$ small, it follows from (3.3)-(3.5) and (3.9) that

$$
\begin{aligned}
& \tau(\xi)-\tau\left(\xi^{\prime}\right) \sim \mu \rho(\bar{\xi}), \\
& T(\xi)-T\left(\xi^{\prime}\right) \sim \mu \tau(\bar{\xi})^{2}+\frac{1}{4} \mu^{3}\left[\tau^{\prime}(\bar{\xi})^{2}+\tau(\bar{\xi}) \tau^{\prime \prime}(\bar{\xi})\right], \text { (5.4) } \\
& U(\xi)-U\left(\xi^{\prime}\right) \sim \mu \tau(\bar{\xi})+\frac{1}{8} \mu^{3} \tau^{\prime \prime}(\bar{\xi}),
\end{aligned}
$$

and hence

$$
W\left(\xi, \xi^{\prime}\right) \sim \frac{1}{12} \mu^{3} \rho(\bar{\xi})^{2} .
$$

Therefore, in the vicinity of $\zeta=1, \bar{k}(\zeta)$ is approximately given by

$$
\begin{aligned}
\vec{K}(\xi) & \sim \frac{1}{4} \Gamma\left(\frac{2}{3}\right)\left(\frac{2 X}{1-X}\right)^{-2 / 3} \int_{-\infty}^{\infty} d \vec{\xi} \int_{-1}^{1} d \mu|\mu| \rho(\bar{\xi})^{2} e^{-i \pi(\operatorname{sg} \mu) / 6} \\
& {\left[\frac{1}{12}|\mu|^{3} \rho(\vec{\xi})^{2}\right]^{-1+5 / 3} } \\
& \sim \Gamma\left(\frac{2}{3}\right)\left(\frac{4 X}{1-X}\right)^{-2 / 3} 3^{7 / 6} \frac{1}{5-1} \int_{-\infty}^{\infty} d \bar{\xi}[\rho(\bar{\xi})]^{2 / 3}
\end{aligned}
$$

and the residue $R_{1}$ is

$$
R_{1}=\Gamma\left(\frac{2}{3}\right)\left(\frac{4 X}{1-X}\right)^{-2 / 3} 3^{7 / 6} \int_{-\infty}^{\infty} d \bar{\xi}[\rho(\bar{\xi})]^{2 / 3} .
$$

One recognizes here several of the factors entering the expression for radiation "during bunch crossing" [11,18]. 


\section{6. - ANALYTIC CONTINUATION INTO THE REGION $1>\operatorname{Re} \zeta>0$}

The considerations of Section 5 indicate that $\bar{\xi}$ and $\mu$ of (5.2) are more appropriate variables. In terms of these variables, the $\bar{K}(\zeta)$ of (4.11) is

$$
\begin{aligned}
\vec{K}(5)= & (3+\zeta)^{-1} \Gamma(1-5 / 3)\left(\frac{2 X}{1-X}\right)^{-1+5 / 3} \int_{-\infty}^{\infty} d \vec{\xi} \int_{-\infty}^{\infty} d \mu|\mu|^{-1}[\tau(\bar{\xi}+\mu / 2)-\tau(\bar{\xi}-\mu / 2)]^{2} \\
& e^{i \beta \mu} e^{-i \pi(5 / 6) s g \mu}|\tilde{W}(\xi, \mu)|^{-1+5 / 3},
\end{aligned}
$$

where

$$
\tilde{W}(\vec{\xi}, \mu)=W\left(\xi, \xi^{\prime}\right)
$$

is an odd function of $\mu$ and has the property from (5.6) that

$$
\tilde{W}(\bar{\xi}, \mu) \sim \frac{1}{12} \mu^{3} \rho(\bar{\xi})^{2}
$$

for $\operatorname{smal1} \mu$.

Before we can study the behaviour of $\overline{\mathrm{K}}(\zeta)$ in the vicinity of $\zeta=0$, we must rewrite the xight-hand side of $(6.1)$ in such a way that it makes sense in that vicinity. This can be accomplished in a variety of ways; we choose one that facilitates furthex analytic continuation to near $\zeta=-1$. Our procedure involves rewriting the $\mu$ integral using suitable contours. We assume that $\rho(\xi)$ is analytic so that analytic continuation to complex values of $\mu$ is possible, at least for sma11 values of $|\operatorname{Im} \mu|$.

Consider the following factor in the integxand of (6.1):

$$
\begin{aligned}
& L(\mu)=|\mu|^{-1} e^{-i \pi(5 / 6) 5 g \mu}|\tilde{W}(\bar{\xi}, \mu)|^{-1+5 / 3} \\
& = \begin{cases}\mu^{-1} e^{-i \pi 5 / 6}[\tilde{W}(\bar{\xi}, \mu)]^{-1+5 / 3} & \text { for } \mu>0, \\
-\mu^{-1} e^{i \pi 5 / 6}[-\tilde{W}(\bar{\xi}, \mu)]^{-1+5 / 3} & \text { for } \mu<0 .\end{cases}
\end{aligned}
$$

Starting with the above expression for $\mu>0$, we continue it analytically to $\mu<0$. The results are, using $(6.3)$,

$$
L\left(\mu e^{i \pi}\right)=\mu^{-1} e^{5 i \pi 5 / 6}[\tilde{W}(\vec{\xi}, \mu)]^{-1+5 / 3}
$$

and 


$$
L\left(\mu e^{-i \pi}\right)=\mu^{-1} e^{-7 i \pi 5 / 6}[\tilde{W}(\vec{\xi}, \mu)]^{-1+5 / 3} .
$$

We attempt to write the $L(-\mu)$, as defined by (6.4), as a linear combination of the two different contributions given by $(6.5)$ and $(6.6)$

$$
L(-\mu)=C_{1} L\left(\mu e^{i \pi}\right)+C_{2} L\left(\mu e^{-i \pi}\right),
$$

where the sum of $C_{1}$ and $C_{2}$ should be 1 :

$$
C_{1}+C_{2}=1
$$

Since $\widetilde{W}(\bar{\xi}, \mu)$ is an odd function of $\mu$, it follows from $(6.4)-(6.7)$ that

$$
C_{1} e^{2 i \pi 5 / 3}+C_{2} e^{-i \pi 5 / 3}=1
$$

Equations (6.8) and (6.9) give

and

$$
C_{1}=e^{-i \pi 5 / 3} \frac{\sin (2 \pi 5 / 3)}{\sin \pi 5}
$$

$$
C_{2}=e^{2 i \pi 5 / 3} \frac{\sin (\pi 5 / 3)}{\sin \pi 5} .
$$

Note that $C_{1}$ and $C_{2}$ are finite as $\zeta \rightarrow 0$, but not for $\zeta \rightarrow-1$. This property turns out to be 1mportant.

Using (6.7) togethex with $(6.10)$, we can rewrite $(6.1)$ in the form

$$
\begin{gathered}
\bar{K}(\zeta)=(3+5)^{-1} \Gamma(1+5 / 3)\left(\frac{2 X}{1-X}\right)^{-1+5 / 3} \int_{-\infty}^{\infty} d \bar{\xi} \int_{C} d \mu \mu^{-1}[\tau(\vec{\xi}+\mu / 2)-\tau(\bar{\xi}-\mu / 2)]^{2} \\
e^{i \beta \mu} e^{-i \pi 5 / 6}[\tilde{W}(\bar{\xi}, \mu)]^{-1+5 / 3},
\end{gathered}
$$

whexe, for the $\mu$ integration, $\int_{C}$ is defined by the weighted average of two contour integrations

$$
\int_{C}=e^{-i \pi 5 / 3} \frac{\sin (2 \pi 5 / 3)}{\sin \pi 5} \int_{C_{+}}+e^{2 i \pi 5 / 3} \frac{\sin (\pi 5 / 3)}{\sin \pi 5} \int_{C_{-}}
$$

In (6.12), the contours $C_{+}$and $C_{-}$of integrations axe along the real axis except for an indentation at the origin into the upper and lower half planes respectively, as shown in Fig. 2 .

Equation (6.11) gives the desired analytic continuation of $\bar{K}(\zeta)$ into the enlarged region 


$$
3>\operatorname{Re} 5>0
$$

except for the pole at $\zeta=1$ already studied in Section 5 .

We can now proceed to the study of the singularity at $\zeta=0$.

\section{7. - RESIDUE OF $\overline{\mathrm{K}}(\zeta)$ AT $\zeta=0$}

While the residue of $\overline{\mathrm{K}}(\zeta)$ at $\zeta=1$ comes from the region of integration in the vicinity of $\xi=\xi^{\prime}$, that at $\zeta=0$ comes from the region of large $|\bar{\xi}|$.

Having in mind the hyperbolic secant distribution, we assume more generally that $\rho(\xi)$ decreases exponentially as $\xi \rightarrow \infty$ and $\xi \rightarrow-\infty$. More explicitly, let

$$
\rho(\xi) \sim\left\{\begin{array}{l}
c_{1} e^{a_{1} \xi}, \text { as } \xi \rightarrow-\infty, \\
c_{2} e^{-a_{2} \xi}, \text { as } \xi \rightarrow \infty,
\end{array}\right.
$$

where $a_{1}, a_{2}, c_{1}$ and $c_{2}$ are four positive constants. By a translation on $\xi$, the values of $c_{1}$ and $c_{2}$ can be made equal; however, such a choice has no particular advantage.

Similar to (5.1), let $\mathrm{R}_{\mathrm{o}}$ be the residue of $\overrightarrow{\mathrm{K}}(\zeta)$ at $\zeta=0$, i.e.,

$$
R_{0}=\lim _{\zeta \rightarrow 0} \zeta \bar{K}(\zeta) \text {. }
$$

In this section, we calculate $R_{0}$.

Let $\tau_{1}$ and $\widetilde{W}_{1}$ be the $\tau$ and $\tilde{W}$ corresponding to

$$
P_{1}(\xi)=c_{1} e^{a_{1} \xi},
$$

while $\tau_{2}$ and $\widetilde{W}_{2}$ to

$$
P_{2}(\xi)=c_{2} e^{-a_{2} \xi} \text {. }
$$

Then from (6.11), near $\zeta=0, \overline{\mathrm{K}}(\zeta)$ is

$$
\bar{K}(5) \sim \bar{K}_{1}(5)+\bar{K}_{2}(5) \text {. }
$$


where

$$
\begin{gathered}
\bar{K}_{1}(\xi)=\frac{1-X}{6 X} \int_{-\infty}^{0} d \bar{\xi}\left(\frac{2}{3} \int_{C_{+}}+\frac{1}{3} \int_{C_{-}}\right) d \mu \mu^{-1}\left[\tau_{1}(\bar{\xi}+\mu / 2)-\tau_{1}(\bar{\xi}-\mu / 2)\right]^{2} \\
e^{i \beta \mu}\left[\tilde{W}_{1}\left(\bar{\xi}_{\xi}, \mu\right)\right]^{-1+5 / 3}
\end{gathered}
$$

and

$$
\begin{gathered}
\bar{K}_{2}(\zeta)=\frac{1-X}{6 X} \int_{0}^{\infty} d \bar{\xi}\left(\frac{2}{3} \int_{C_{+}}+\frac{1}{3} \int_{C_{-}}\right) d \mu \mu^{-1}\left[\tau_{2}(\bar{\xi}+\mu / 2)-\tau_{2}(\bar{\xi}-\mu / 2)\right]^{2} \\
e^{i \beta \mu}\left[\tilde{W}_{2}(\bar{\xi}, \mu)\right]^{-1+5 / 3}
\end{gathered}
$$

Since $\overline{\mathrm{K}}_{2}(\zeta)$ can be obtained from $\overline{\mathrm{K}}_{1}(\zeta)$ by the replacements $a_{1} \rightarrow a_{2}$ and $c_{1} \rightarrow c_{2}$, it is sufficient to concentrate on $\overline{\mathrm{K}}_{1}(\zeta)$.

From (3.3)-(3.5) and (3.9), the $\rho_{1}$ of (7.3) leads to

$$
\begin{aligned}
& \tau_{1}(\xi)=\left(c_{1} / a_{1}\right) e^{a_{1} \xi}, \\
& T_{1}(\xi)=\frac{1}{2}\left(c_{1}^{2} / a_{1}^{3}\right) e^{2 a_{1} \xi}, \\
& U_{1}(\xi)=\left(c_{1} / a_{1}^{2}\right) e^{a_{1} \xi},
\end{aligned}
$$

and

$$
W_{1}\left(\xi, \xi^{\prime}\right)=\frac{c_{1}^{2}}{2 a_{1}^{3}}\left[e^{2 a_{1} \xi}-e^{2 a_{1} \xi^{\prime}}-\frac{2}{a_{1}\left(\xi-\xi^{\prime}\right)}\left(e^{a_{1} \xi}-e^{a_{1} \xi^{\prime}}\right)^{2}\right] .
$$

The important point here is that the right-hand side of (7.11) is of the form of a product $e^{2 a \xi}$ times a function of $\mu$. Therefore the $\bar{\xi}$ integration in (7.6) can be carried out eastly, leading to

$$
\bar{K}_{1}(\zeta) \sim \zeta^{-1} \frac{1-X}{2 X}\left(\frac{2}{3} \int_{C_{+}}+\frac{1}{3} \int_{C_{-}}\right) d \mu e^{2 i \beta \mu / a_{1}} \frac{\sinh \mu}{\mu \cosh \mu-\sinh \mu} \text { (7.12) }
$$

for $\zeta$ small. This exhibits explicitly the pole structure at $\zeta=0$. Since the value of $c_{1}$ can be changed by shifting $\xi$, it cannot appeax in the residue at $\zeta=0$; indeed it does not.

Even for small $\beta$, the integral on the right-hand side of (7.12) cannot be carried out explicitly. We define for this purpose a function 


$$
E(\beta)=\left(\frac{2}{3} \int_{C_{+}}+\frac{1}{3} \int_{C_{-}}\right) d \mu e^{i \beta \mu} \frac{\sinh \mu}{\mu \cosh \mu-\sinh \mu},
$$

which is studied in Appendix A. The residue of $\overline{\mathrm{K}}(\zeta)$ at $\zeta=0$ is then

$$
R_{0}=\frac{1-X}{2 X}\left[E\left(\frac{2 \beta}{a_{1}}\right)+E\left(\frac{2 \beta}{a_{2}}\right)\right] \text {. }
$$

This gives the contribution to the photon spectrum which can be associated with the ends of the bunch.

When $L_{e} \gg L_{b}$, this function $E$ includes a logarithmic contribution.

\section{8. - ANALYTIC CONTINUATION INTO THE REGION $0>\operatorname{Re} \zeta>-1$}

In order to solve the problem of the effect of density gradient and the mystery of the divergent integral when the results of [8-11] are applied to a distribution such as (1.1), we need to study the behaviour of $\overrightarrow{\mathrm{K}}(\zeta)$ near $\zeta=-1$. For this purpose, we must rewrite the right-hand side of (6.11) in such a way that it makes sense in that vicinity. Similax to the development of Section 6, the method of deforming the contour of integration is to be used. Unlike that of section 6, this deformation is applied to the $\bar{\xi}$ integration.

First we assume that the domain of analyticity in $\bar{\xi}$ is large enough to permit the deformation of the contour. However, the result to be obtained in Section 9 can be written in a way that this analyticity is not essential. Since the singularity of $\overline{\mathrm{K}}(\zeta)$ at $\zeta=0$ is a pole, not a branch point, and the result (7.14) on $\mathrm{R}_{\mathrm{o}}$ is of the form of two separate contributions from the two ends of the bunch, the analytic continuation can be carried out via either the upper half plane or the lowex half plane.

The required analytic continuation is performed by merely rewriting (6.11) in the form

$$
\begin{gathered}
\bar{K}(\xi)=(3+\zeta)^{-1} \Gamma(1-\zeta / 3)\left(\frac{2 X}{1-X}\right)^{-1+\zeta / 3} \int_{b} d \bar{\xi} \int_{C} d \mu \mu^{-1}[\tau(\vec{\xi}+\mu / 2)-\tau(\bar{\xi}-\mu / 2)]^{2} \\
e^{i \beta \mu} e^{-i \pi \zeta / 6}[\tilde{W}(\bar{\xi}, \mu)]^{-1+\zeta / 3}
\end{gathered}
$$

where the contour $G$ can be, for example, any of the four shown in Fig. 3. Except for the simple poles at $\zeta=1$ and $\zeta=0$, Eq. (8.1) is valid in the furthex enlarged region 


$$
3>\operatorname{Re} 5>-1 \text {. }
$$

\section{9. - RESIDUE OF $\overline{\mathrm{K}}(\zeta)$ at $\zeta=-1$}

Once again, $\overline{\mathrm{K}}(\zeta)$ has a simple pole at $\zeta=-1$. Similar to (5.1) and (7.2), let $\mathrm{R}_{-1}$ be the residue

$$
R_{-1}=\lim _{\zeta \rightarrow-1}(\zeta+1) \bar{K}(\zeta)
$$

The origin of this pole is exceptionally simple: for $\zeta$ near -1 , the $\mu$ integxation is approximately, by $(6.12)$,

$$
\begin{aligned}
\int_{C} & \sim-\frac{\sqrt{3}}{2 \pi(\zeta+1)} e^{i \pi / 3}\left[\int_{C_{-}}-\int_{C_{+}}\right] \\
& =-\frac{\sqrt{3}}{2 \pi(\zeta+1)} e^{i \pi / 3} \int_{C_{0}},
\end{aligned}
$$

where $C_{0}=C_{-}+C_{+}$is a small counter-clockwise circle around the origin. Therefore, by ( 8.1$)$, the residue is

$$
\begin{gathered}
R_{-1}=\frac{-i \sqrt{3}}{4 \pi} \Gamma\left(\frac{4}{3}\right)\left(\frac{2 X}{1-X}\right)^{-4 / 3} \int_{b} d \bar{\xi} \int_{C_{0}} d \mu \mu^{-1}[\tau(\bar{\xi}+\mu / 2)-\tau(\bar{\xi}-\mu / 2)]^{2} \\
e^{i \beta \mu}[\tilde{W}(\bar{\xi}, \mu)]^{-4 / 3}
\end{gathered}
$$

The procedure of [11] applies, and the result is

$R_{-1}=\frac{2}{15} \Gamma\left(\frac{4}{3}\right)\left(\frac{4 X}{1-X}\right)^{-4 / 3} 3^{5 / 6} \int_{b} d \bar{\xi} \rho(\bar{\xi})^{-8 / 3}\left[3 \rho(\bar{\xi}) \rho^{\prime \prime}(\bar{\xi})-4 \rho^{\prime}(\vec{\xi})^{2}\right] .(9.4)$

This differs fxom the previously obtained results [8-11] in an essential way, namely the contour of integration is not the real axis, but, for example, any of the paths shown in Fig. 3. With such a path of integration, the integral is we11-defined, and no cut-off is needed.

In some circumstances, it is possible to deform the contour $b$ back to the real axis at the expanse of subtracting and adding some terms. For example, if the condition ( 7.1 ) is strengthened to be 


$$
\rho(\xi)= \begin{cases}c_{1} e^{a_{1} \xi}+0\left(e^{5 a_{1} \xi / 3}\right), & \text { as } \xi \rightarrow-\infty, \\ c_{2} e^{-a_{2} \xi}+0\left(e^{-5 a_{2} \xi / 3}\right), & \text { as } \xi \rightarrow \infty,\end{cases}
$$

then the subtraction of the integrand calculated on the basis of the leading behavioux makes it possible to use the real axis as the contour of integration. Such a procedure makes it unnecessary to assume analyticity in $\bar{\xi}$. However, the resulting formula for $R_{-1}$ is not elegant and also not especially informative.

Equation (9.4) can be simplified slightly by an integration by parts. Because of the contour $G$, the integrated terms are zexo and the result is

$$
R_{-1}=\frac{2}{15} \Gamma\left(\frac{4}{3}\right)\left(\frac{4 X}{1-X}\right)^{-4 / 3} 3^{5 / 6} \int_{b} d \xi \rho(\xi)^{-8 / 3} \rho^{\prime}(\xi)^{2} \text {. }
$$

Although the integrand is positive on the real axis, $R_{-1}$ may be positive ox negative.

\section{0. - HYPERBOLIC SECANT DISTRIBUTION}

As an example, we apply our results to the hyperbolic secant distribution $(1.1)$ :

$$
\begin{aligned}
\rho(\xi) & =\frac{1}{4} \operatorname{sech}^{2}(\xi / 2) \\
& =\frac{e^{\xi}}{\left(1+e^{\xi}\right)^{2}} .
\end{aligned}
$$

The factor $1 / 4$ is introduced so that $\rho(\xi)$ is properly normalized. Comparison with (7.1) shows that

$$
a_{1}=a_{2}=1
$$

It follows from $(5.8),(7.14)$ and $(9.6)$ that, for the present case,

$$
\begin{aligned}
& R_{1}=\frac{1}{2 \pi}\left[\frac{81(1-X)}{4 X}\right]^{2 / 3}\left[\Gamma\left(\frac{2}{3}\right)\right]^{4}, \\
& R_{0}=\frac{1-X}{X} E\left(\frac{2 X}{1-X} \frac{L_{6}}{L_{e}}\right),
\end{aligned}
$$

and 


$$
R_{-1}=-\frac{1}{5 \pi}\left(\frac{1-x}{4 x}\right)^{4 / 3} 3^{1 / 3}\left[\Gamma\left(\frac{1}{3}\right)\right]^{4} .
$$

Since $R_{-l}$ is negative, contrary to previous conjecture $[8,9]$, it may be worthwhile to give a derivation of (10.5). For the distribution (10.1), the $R_{-1}$ of $(9.6)$ is

$$
R_{-1}=\frac{2}{15} \Gamma\left(\frac{4}{3}\right)\left(\frac{1-x}{4 X}\right)^{4 / 3} 3^{5 / 6} I_{-1}
$$

where

$$
I_{-1}=\int_{b} d \xi\left[\frac{e^{\xi}}{\left(1+e^{\xi}\right)^{2}}\right]^{-8 / 3}\left[\frac{\left(1-e^{\xi}\right) e^{\xi}}{\left(1+e^{\xi}\right)^{3}}\right]^{2} .
$$

Define a function of a complex variable $\eta$ by

$$
I_{-1}(\eta)=\int_{b} d \xi\left[\frac{e^{\xi}}{\left(1+e^{\xi}\right)^{2}}\right]^{-2+\eta}\left[\frac{\left(1-e^{\xi}\right) e^{\xi}}{\left(1+e^{\xi}\right)^{3}}\right]^{2} \text {. }
$$

then $I_{-1}(\eta)$ has a simple pole at $\eta=0$, and the desired $I_{-1}$ is

$$
I_{-1}=I_{-1}(-2 / 3)
$$

For $\operatorname{Re} \eta>0$, the contoux $b$, as shown for example in Fig. 3, can be replaced by the real axis, and thus the evaluation of $I_{-l}(\eta)$ is straightforward:

$$
I_{-1}(\eta)=\frac{2}{\eta} \frac{[\Gamma(1+\eta)]^{2}}{\Gamma(2+2 \eta)} \text {. }
$$

This shows explicitly the pole structure at $\eta=0$, and also that it is positive for $\eta>0$. However, analytic continuation to negative values of $\eta$ gives

$$
I_{-1}=I_{-1}(-2 / 3)=-3[\Gamma(1 / 3)]^{2} / \Gamma(2 / 3) \text {, }
$$

which is negative. The lesson is that we have to be very careful in providing reliable arguments about even the sign of higher-order corrections.

\section{1. - SUMMARY AND DISCUSSIONS}

When the corxelation length $\ell_{c}$ is much shorter than the nominal bunch length $L_{b}$, the energy spectrum of the beamstrahlung photon is given approximately by

$$
I(X) \sim \frac{\alpha}{2 \pi} \frac{2-2 X+X^{2}}{1-X}\left[\frac{L_{b}}{l_{c}} R_{1}+R_{0}+\frac{l_{c}}{L_{b}} R_{-1}\right]
$$


where $R_{1}, R_{0}$ and $R_{-1}$ are given by Eqs. (5.8), (7.14) and (9.6) respectively. The extra factor present in the Dixac case has been explicitly written down.

It is instructive to rewrite this result in terms of the actual charge density $P_{o}(z)$ xather than the normalized charge density $p\left(z / L_{b}\right)$. They axe related by $(3.1)$.

$$
\begin{aligned}
I(X) & \sim \frac{\alpha}{2 \pi} \frac{2-2 X+X^{2}}{1-X}\left\{\Gamma\left(\frac{2}{3}\right)\left(\frac{1-X}{4 X}\right)^{2 / 3} 3^{7 / 6}\left(\frac{\pi^{2} R^{2}}{k_{i}}\right)^{1 / 3} \int_{-\infty}^{\infty} d z\left[e \rho_{0}(z)\right]^{2 / 3}\right. \\
& +\frac{1-X}{2 X}\left[E\left(\frac{2 X}{1-X} \frac{1}{a_{10} L_{e}}\right)+E\left(\frac{2 X}{1-X} \frac{1}{a_{20} L_{e}}\right)\right] \\
& \left.+\frac{2}{3} \Gamma\left(\frac{4}{3}\right)\left(\frac{1-X}{4 X}\right)^{4 / 3} 3^{-1 / 6}\left(\frac{k_{i}}{\pi^{2} R^{2}}\right)^{1 / 3} \int_{6} d z\left[e \rho_{0}(z)\right]^{-8 / 3}\left[e \rho_{0}^{\prime}(z)\right]^{2}\right\},
\end{aligned}
$$

where, by (7.1),

$$
a_{10}=\lim _{z \rightarrow-\infty} P_{0}^{\prime}(z) / P_{0}(z)
$$

and

$$
a_{20}=-\lim _{z \rightarrow \infty} p_{0}^{\prime}(z) / p_{0}(z) \text {. }
$$

Examples of the contour of integration axe shown in Fig. 3. The important point to be noticed in Eq. (11.2) is that the nominal bunch length $\mathrm{L}_{b}$ does not appear. anywhere on the right-hand side. This is the way it should be, because there is nothing to prevent us from using $2 \mathrm{~L}_{b}$ or $\frac{1}{2} \mathrm{~L}_{b}$, for example, instead of $\mathrm{L}_{b}$ in all the intermediate steps.

For the special case of the hyperbolic secant distribution

$$
P_{0}(z)=\frac{N e}{\pi L_{b} R^{2}} \frac{e^{z / L_{b}}}{\left(1+e^{z / L_{b}}\right)^{2}},
$$

the photon spectrum is given by (11.1) together with Eqs. (10.3)-(10.5).

We conclude the present paper with the following comments.

(A) The result (11.1), or equivalently (11.2), takes the form of the sum of three terms. Roughly speaking, the first term gives the beamstrahlung during beam crossing. The second term gives the effects of the bunch ends, and is especially large when $\mathrm{L}_{e} \gg \mathrm{L}_{b}$, as seen from the asymptotic behaviour (A6). In this case of $\mathrm{L}_{e} \gg \mathrm{L}_{b}$, this second term consists of two pieces, one of which is logarithmic. This logarithmic piece is similar to the one found for the uniform bunch [1], and can become sizeable for very high energies.

(B) The value of the third term depends on the variation of charge densities $\rho_{0}^{\prime}(z)$ and $\rho_{0}^{\prime \prime}(z)$ along the bunch. Contrary to the claims of Chen and Yokoga $[8,9]$, it is 
not large. At least for the special case of the hyperbolic secant bunch (11.4), this term is negative, reducing slightly the beamstrahlung energy loss.

(C) In obtaining the present result, we have assumed an exponential decrease in the charge density far away from the centre of the bunch. This is as good a description as, if not better than, a Gaussian bunch for realistic cases. Nevertheless, it may be asked what the corresponding result looks like for a Gaussian bunch. Unfortunately, the answer is that it is very much more complicated, and hence probably of little practical use.

\section{ACKNOWLEDGEMENTS}

We are grateful to Jochen Bartels, John Be11, Toishiro Kinoshita and Kxzysztof Kurek for helpful discussions. We are indebted to Kurt korbig for the numerical calculation of the function $E(\beta)$. One of us (T.T.W.) wishes to thank the CERN Theory Division for its kind hospitality. 


\section{APPENDIX}

In this appendix, we study some of the properties of the $E(\beta)$ defined by Eq. (7.13). Since $\beta$ is positive, we can close the contour of integration in the upper half plane to get

$$
E(\beta)=-2 \pi\left[\beta-\sum_{n=1}^{\infty} \frac{e^{-\beta y_{n}}}{y_{n}}\right],
$$

where $y_{n}$ is the $n^{\text {th }}$ positive zero of

$$
y-\tan y=0
$$

The first few zeros are at [19]

$$
\begin{aligned}
& y_{1}=4.493409458, \\
& y_{2}=7.725251838, \\
& y_{3}=10.90412166,
\end{aligned}
$$

etc. From (A1) it is clear that $E(\beta)$ is a decreasing function of $\beta$ for positive $\beta$.

It remains to write down explicitly the behaviour of $E(\beta)$ for small positive values of $\beta$. For large $n, y_{n}$ is asymptotically given by

$$
y_{n} \sim\left(n+\frac{1}{2}\right) \pi-\left[\left(n+\frac{1}{2}\right) \pi\right]^{-1}
$$

We know that [20]

$$
\begin{aligned}
\sum_{n=0}^{\infty} & \left(n+\frac{1}{2}\right)^{-1} e^{-\beta \pi n}=\Phi\left(e^{-\beta \pi}, 1, \frac{1}{2}\right) \\
& =2{ }_{2} F_{1}\left(1, \frac{1}{2} ; \frac{3}{2} ; e^{-\beta \pi}\right) \\
& \sim h_{0}^{\prime \prime}-\ln \left(1-e^{-\beta \pi}\right) \\
& \sim 2 \ln 2-\ln (\beta \pi),
\end{aligned}
$$

where $\Phi$ is defined on p. 27 [Eq. (1)], ${ }_{2} F_{1}$ is the hypergeometric function [p. 30, Eq. (10)], $h_{0}$ is defined on p. 110 [Eqs. (12) and (13)], all of Ref. [20]. Therefore

$$
E(\beta)=2\left(-\ln \beta+K_{e}\right)+O(\beta)
$$

as $\beta \rightarrow 0^{+}$, where $\mathrm{K}_{\mathrm{e}}$ is defined by

$$
K_{e}=-\ln \pi+2 \ln 2-2+\sum_{n=1}^{\infty}\left(\frac{\pi}{y_{n}}-\frac{2}{2 n+1}\right)
$$




$$
=-1.71315032 \text {. }
$$

The function $E(\beta)$ is plotted in Fig. 4 . 


\section{REFERENCES}

[1] M. Jacob and T.T. Wu, Phys.Lett. B197 (1987) 253.

[2] M. Jacob and T.T. Wu, Nuc1.Phys. B303 (1988) 373.

[3] M. Jacob and T.T. Wu, Nucl.Phys. B303 (1988) 389.

[4] T. Himel and J. Siegrist, AIA Conference Proceedings 130 (1985); R.J. Noble, SLAC-PUB 3871 (1986).

[5] M. Tignex, Nuovo Cimento 37 (1965) 1228;

B. Richtex, Nuc1.Inst. and Methods 136 (1976) 47 ;

B. Richter, Proceedings of the 1984 ICFA Seminar on Future Perspective in High Energy Physics;

U. Ama1di, Phys.Lett. 61B (1976) 313;

U. Amaldi, "Introduction to the Next Generation of Linear Colliders", CERN EP 87-169 (1987).

[6] R. Blankenbeclex and S.D. Dre11, Phys.Rev. B36 (1987) 277.

[7] M. Bell and J.S. Be11, CERN PS 87-53 (1987);

M. Bell and J.S. Bell, CERN TH 4936 (1987).

[8] P. Chen, SLAC-PUB-4391 (1987);

[9] P. Chen and K. Yokoga, SLAC-PUB-4597 (1988).

[10] M. Bell and J.S. Bell, CERN TH 5056 (1988).

[11] M. Jacob and T.T. Wu, CERN TH 5133 (1988).

[12] B. Autin, CERN PS, private communication.

[13] L.I. Schiff, Phys.Rev. 103 (1956) 443;

D.S. Saxon, Phys.Rev. 107 (1957) 871;

H. Cheng and T.T. Wu in High Energy Collisions, ed. C.N. Yang (Gordon and Breach, 1969) 329.

[14] T.T. Wu, Phys.Rev. 108 (1957) 466;

D.S. Saxon and L.I. Schiff, Nuovo Cimento 6 (1957) 614;

V.P. Maslov and M.V. Fedoriuk, Semi-Classical Approximation in Quantum Mechanics (D. Reidel Publ. Co., 1981) Ch. 14.

[15] J.D. Bjorken and T.T. Wu, Phys.Rev. 130 (1963) 2566;

[16] P. Osland and T.T. Wu, Nucl.Phys. B288 (1987) 77 and 95.

[17] H. Cheng and T.T. Wu, Expanding Protons, MIT Press (1987);

[18] R. Blankenbecler and S.D. Drel1, SLAC-PUB-4483 (1987).

[19] National Bureau of Standards, Handbook of Mathematical Functions, eds. M. Abramowitz and I.A. Stegun (1964), p. 224 .

[20] Bateman Manuscript Project, Higher Transcendental Functions, ed. A. Erdelyi (McGraw Hill Book Company, 1953), Vol. I. 
FIGURE CAPTIONS

Fig. 1 Feynman diagram for $e^{-}+$bunch $\rightarrow e^{-}+\gamma+$ bunch.

Fig. 2 The contours $c_{+}$and $c_{-}$of integration in the $\mu$ plane.

Fig. 3 Four possible choices of the contour $b$ of integration. The horizontal straight portion of the four contours is on the real axis.

Fig. 4 The function $E(\beta)$ that describes end effect in beamstrahlung. 


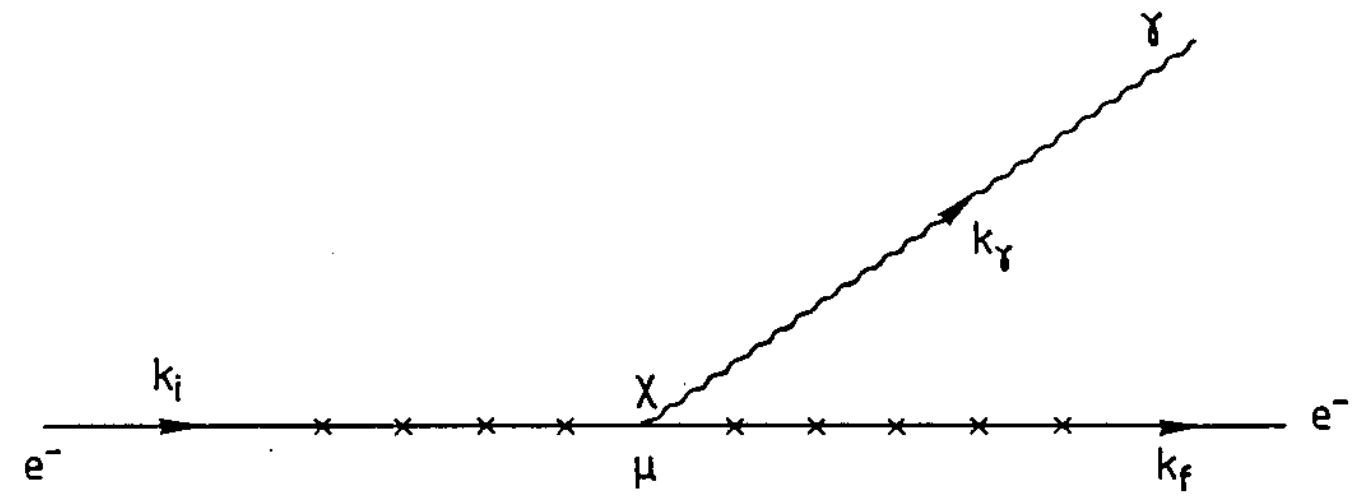

- Figure 1 -
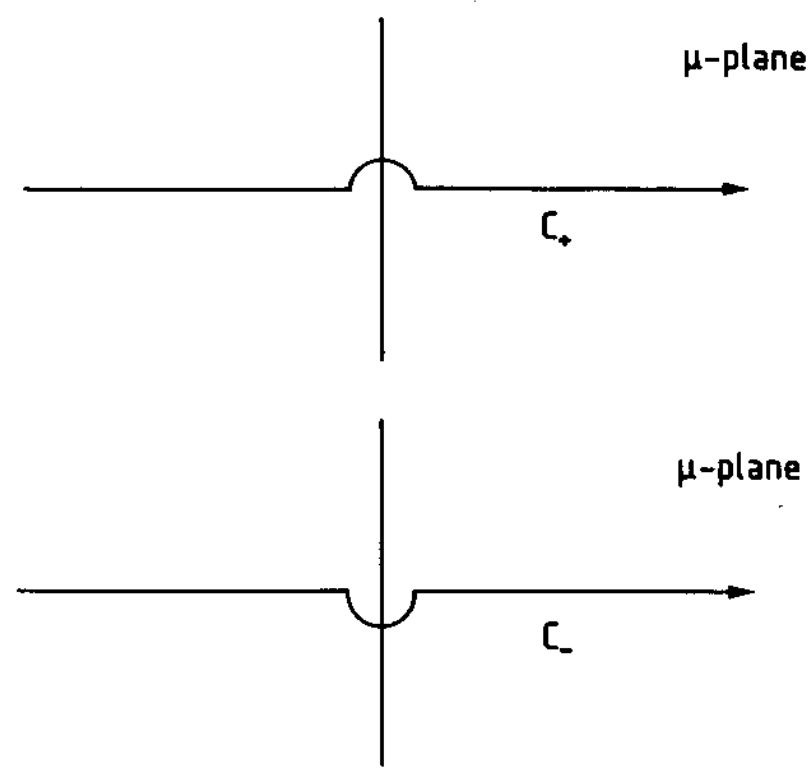

- Figure 2 - 


$$
{ }_{3} 8
$$




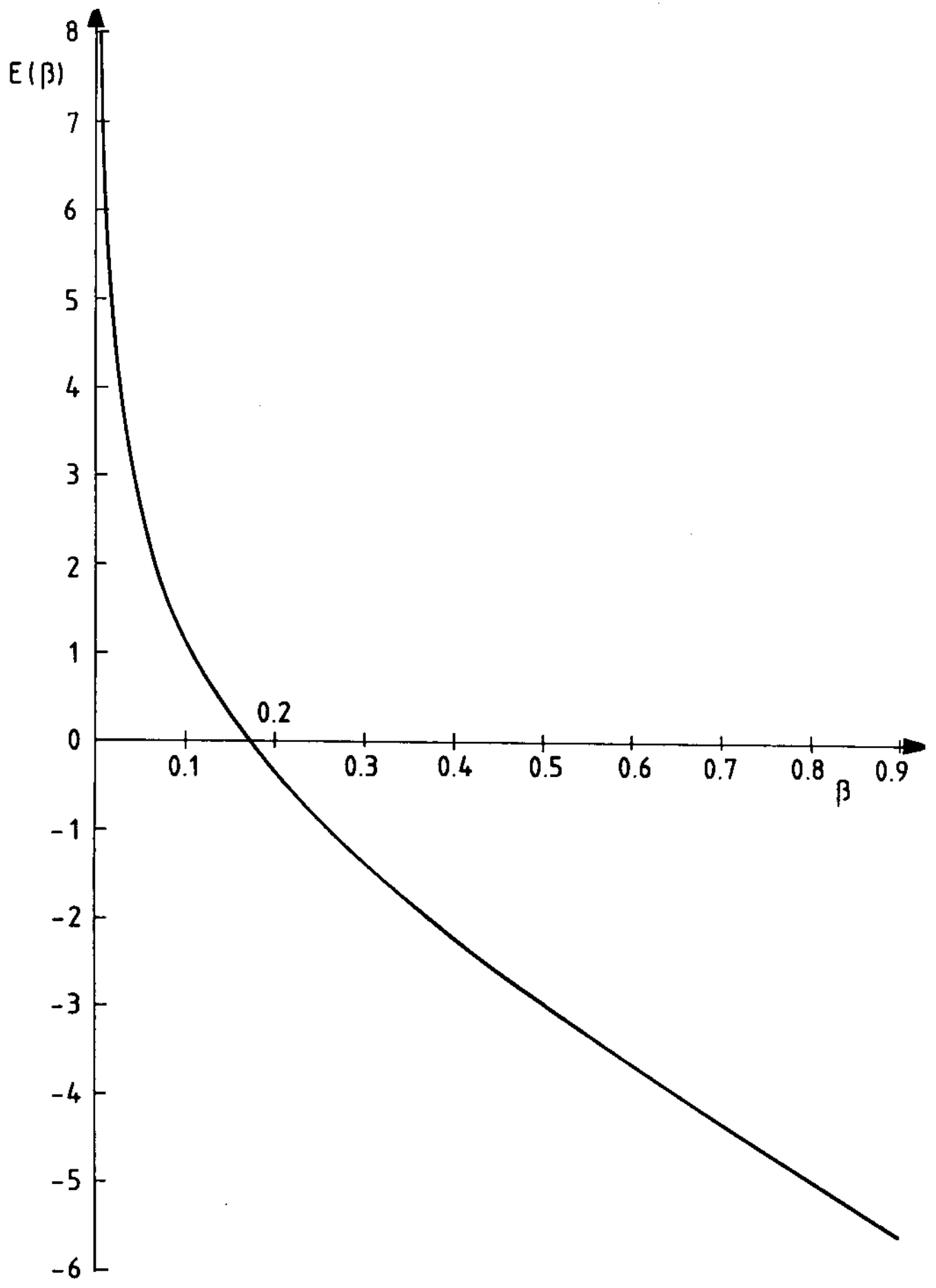

- Fig. 4 - 